\title{
Propiedades psicométricas de la Escala para medir el Disfrute Experimentado en Niños y Adolescentes (EDENA) en población de Michoacán (México)
}

\section{Psychometric properties of the Scale to measure the Enjoyment Experienced by Children and Adolescents) (SEECA) in Michoacan population (Mexico)}

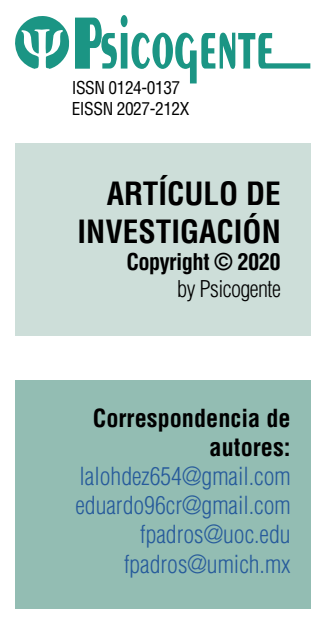

Recibido: 20/01/19 Aceptado: $12 / 08 / 19$ Publicado: $01 / 01 / 20$

\author{
Eduardo Gómez Hernández (iD - Eduardo Carrillo Ramírez (iD) \\ Ferran Padrós Blázquez (iD) \\ Universidad Michoacana de San Nicolás de Hidalgo, Morelia, México
}

Resumen

Objetivo: Construir una escala para medir el disfrute experimentado en niños y adolescentes y estudiar sus propiedades psicométricas.

Método: Estudio de tipo instrumental, donde participaron 487 estudiantes de secundaria de edades comprendidas entre 11 y 15 años, que respondieron a la escala objeto de estudio (Escala para medir el Disfrute Experimentado en Niños y Adolescentes, EDENA), junto a las escalas PANASN (Positive And Negative Affect Schedule) para niños y adolescentes elaboradas por Sandín (2003) y la escala de Depresión del Centro de Estudios Epidemiológicos (CES-D) de Radloff (1977) adaptada a población mexicana por Jiménez-Tapia, Wagner, Rivera-Heredia y González-Forteza (2015).

Resultados: A través de un Análisis Factorial Exploratorio se observó que la estructura interna resultó unifactorial. Respecto a la consistencia interna, el valor del alfa de Cronbach de la escala resultó de .650, aceptable teniendo en cuenta que solo tiene cinco reactivos, los cuales mostraron adecuadas propiedades psicométricas. Se realizaron correlaciones de Pearson con otras medidas, obteniendo una correlación baja y positiva con la escala de afecto positivo $(r=.368)$ y correlaciones bajas y negativas con el afecto negativo $(r=-.361)$ y con la sintomatología depresiva $(r=-.179)$.

Conclusiones: Se concluye que la EDENA es instrumento que puede resultar de gran utilidad, ya que podría considerarse un indicador de presencia de anhedonia, que se asocia con disfunción social y emocional, además de psicopatología. La EDENA posee adecuadas propiedades psicométricas (validez de constructo, confiablidad y validez concurrente) para ser administrado en la ciudad de Morelia (México).

Palabras clave: promoción de la salud; desarrollo emocional; bienestar del niño; estudio instrumental; psicometría.

\section{Abstract}

Objective: This paper aims to build a scale for enjoyment measuring in order to measure enjoyment experienced by children and adolescents and also, it aims to study this scale's psychometric properties.

Method: An instrumental approach is conducted in this study ; 487 high school students aged 11 to 15 years old were sampled, answering SEECA (scale to measure the enjoyment experienced by children and adolescents) ; along with this scale, PANAS-C (Positive and Negative Affect Schedule) elaborated by Sandín (2003) and the Epidemiological Studies Center Scale of Depression CES-D of Radloff (1977) adapted to the Mexican population by Jiménez-Tapia, Wagner, Rivera-Heredia and González-Forteza (2015) were also applied to children and adolescents.

Results: Suggest a uni-factorial structure according to an exploratory factor analysis. Based on Chronbach 's alpha's value, the internal consistency, is determined in .650, acceptable taking into account that it has only 5 items, which showed adequate psychometric properties. Pearson's correlation was made with other measurements, obtaining a low positive correlation with the positive affect's scale $(r=.368)$, and low negative correlations with negative affect $(r=-.361)$ and depressive symptoms $(r=-.179)$.

Cómo citar este artículo (APA):

Gómez Hernández, E.; Carrillo Ramírez, E. \& Padrós Blázquez, F. (2020). Propiedades psicométricas de la Escala para medir el Disfrute Experimentado en Niños y Adolescentes (EDENA) en población de Michoacán (México). Psicogente 23(43), 1-18. https://doi.org/10.17081/psico.23.43.3761 
Conclusion: SEECA, is a very useful instrument, since it may recognize the presence of anhedonia, which is associated with social and emotional dysfunction, and also as a psychopathology. The SEECA has adequate psychometric properties (construct validity, reliability and concurrent validity ) to be administrated in Morelia's city (Mexico)

Key words: health promotion; emotional development; child welfare; instrumental study; Psychometrics.

\section{INTRODUCCIÓN}

La Psicología durante la mayor parte de su historia, ha dedicado muchos esfuerzos tratando de identificar, medir y estudiar los elementos que regulan el sufrimiento y el malestar de las personas. Sin embargo, no se han invertido esfuerzos con la misma magnitud en estudiar y medir los factores que pueden regular el disfrute o goce que experimentan las personas (Padrós, 2002). Aproximadamente, desde finales de 1999 y principios de 2000, puede considerarse que surgió la subdisciplina conocida como Psicología Positiva, la cual Castro (2010) concibe como el estudio del funcionamiento psicológico óptimo de las personas, de los grupos y de las instituciones. Lo que vendría a corregir el desequilibrio entre investigación psicológica y práctica centrada exclusivamente en variables psicopatológicas. Por otro lado, según la OMS (1948, citado por Alcántara, 2008) la salud es definida como "un estado de completo bienestar físico, psicológico y social y no la mera ausencia de enfermedad o minusvalía" (p.1). La salud es un recurso de la vida, no su objetivo. Es un concepto positivo que subraya los recursos sociales y personales así como las capacidades físicas. Además, como señalan Bustos y Russo (2018) la salud mental en la infancia y se puede extender a la adolescencia, es un tema de interés nacional e internacional, ya que se ha observado un incremento en la prevalencia de trastornos mentales en esta población.

Por otro lado, desde antes del surgimiento de la Psicología Positiva, autores como Maslow (1968) con su concepto de autorrealización, Rogers (1961) con el de realización plena, Allport (1961) con el de madurez y Diener (1984) con el de bienestar subjetivo, se interesaron por el buen funcionamiento del ser humano. Ryan y Deci (2001) distinguieron organizar el estudio sobre el bienestar tomando como referencia dos antiguas corrientes filosóficas, la hedónica y la eudaimónica, y al que se alude como bienestar subjetivo y como bienestar psicológico respectivamente (Keyes, Ryff \& Shmotkin, 2002).

El bienestar subjetivo es concebido como un constructo compuesto por un elemento cognitivo y dos afectivos (Diener, 1984). El elemento cognitivo es el de satisfacción con la vida que se define como la evaluación realizada por un individuo sobre la calidad global de su propia vida en conjunto (Veenhoven, 
1994). Los dos elementos afectivos son el afecto positivo y el afecto negativo, los cuales son concebidos por separado, aunque todavía persiste cierta controversia teórica respecto a si el afecto positivo y negativo son parte de una sola dimensión bipolar (donde en un polo se encuentra un afecto positivo extremo y en el otro, un afecto negativo extremo), o dos dimensiones unipolares independientes (se refiere al grado de afecto positivo en una dimensión y al nivel de afecto negativo en otra). En la revisión teórica y empírica realizada por Padrós-Blázquez, Navarro-Contreras y Soriano-Más (2012) se señala que el afecto incluye como mínimo dos modalidades afectivas: 1) las emociones que se caracterizan por ser evocadas a partir de una situación estimular, ser específicas, de elevada intensidad, poca duración y por provocar en la persona respuestas fisiológicas notables, predisponiéndola a realizar comportamientos adaptativos; 2 ) el tono afectivo o humor, que es una modalidad afectiva más difusa, no se relaciona con un evento específico, es de baja intensidad, larga duración, y no se asocia a cambios fisiológicos en el organismo. Sobre el humor o tono afectivo parece ser más conveniente conceptualizarse como una sola dimensión unipolar. Sin embargo, respecto a las emociones, los datos ofrecen claramente mayor apoyo al modelo bidimensional unipolar Padrós-Blázquez et al. (2012).

El disfrute se puede definir como un estado afectivo positivo que incluye estados como placer, gusto y diversión (Scanlan \& Symons, 1992). Davis (1982) señaló que un individuo disfruta de algo, si este "algo" (la exposición a una situación particular, la realización de una acción determinada, el contacto con un objeto concreto, etc.) provoca en la persona una serie de creencias relativas a que este "algo" aumenta significativamente el placer (entiéndase que incluye gusto y diversión) que el individuo está experimentando. De modo que el disfrute puede ser concebido como un incremento en el nivel de afecto positivo (respecto a un estado difuso, no relacionado con ninguna circunstancia específica, es decir el humor o tono afectivo en el que se encuentra el individuo) evocado o relacionado con una circunstancia específica, con una intensidad moderada o intensa y de una duración limitada. Por ello, como señala Wankel (1993, como se citó en Kimiecik y Harris, 1996), el disfrute puede ser concebido como una emoción positiva.

Por otro lado, Seligman y Csikszentmihalyi (2000) señalaron que el disfrute (complejo o evolucionado, según nuestra nomenclatura) alude aquellos estados afectivos que la gente experimenta que traspasan la barrera de la homeostasis. Es decir, va más allá del placer (básico o fundamental desde nuestra nomenclatura) derivado de la satisfacción de una necesidad como el 
hambre o la sed (por ejemplo). El disfrute (complejo) puede experimentarse cuando se aprecia (con carga emocional positiva) una competencia atlética, una representación artística, o se realiza un acto altruista, incluso manteniendo una conversación estimulante.

Desde nuestra perspectiva, resulta relevante la distinción entre lo que denominamos placer básico y disfrute complejo o evolucionado. Kringelbach y Berridge (2009) distinguen entre los circuitos neuronales de los placeres fundamentales (placer básico) y los placeres superiores (disfrute complejo), los cuales se solapan, es decir, comparten una base neural común. Consideramos que el disfrute complejo es un mecanismo evolucionado del placer básico de modo congruente con la idea de que los sistemas más evolucionados se asientan en los más antiguos, no sustituyéndose sino solapándose (Allman, 1999). Desde nuestra propuesta, áreas neuroanatómicas del cerebro más antiguo en sentido filogenético y ontogenético, situadas en las zonas más profundas del cerebro (núcleo accumbens, pálido ventral, tronco encefálico) estarían implicadas en el placer básico, y otras que se encuentran en la corteza (orbitofrontal, cingulada, medial prefrontal e insular) y probablemente con la implicación de otras partes del neocórtex, serían el sustrato de las experiencias de disfrute complejo. Puede verse una revisión sobre las bases neuroanatómicas del placer y de la felicidad (donde se incluiría el disfrute complejo) en Kringelbach y Berridge (2009; 2010a; 2010b).

Es importante destacar que son varias las vías por las que un individuo puede obtener placer (básico) y probablemente más las que den lugar a disfrute (complejo). Kringelbach y Berridge (2009) afirman que las rutas del sabor y del olor de los alimentos, las cuales se encuentran entre los placeres fundamentales cuando se relacionan con la necesidad básica, son de las más estudiadas. Aunque si nos referimos a la degustación, por ejemplo de un vino o del deleite de los sabores de los alimentos, entonces el placer obtenido va más allá de satisfacer una necesidad básica, y puede considerarse un disfrute complejo desde nuestra perspectiva (y la vía de esta modalidad carece de investigación en el ámbito de las neurociencias). La ruta del sexo, que en principio también puede considerarse un placer fundamental (aunque en el humano, inciden factores que pueden elevarlo a disfrute complejo), todavía se encuentra en los primeros estadios de desarrollo del conocimiento. Por otro lado, los mismos autores señalan que en los animales sociales, donde se incluyen los humanos, las interacciones con otros especímenes de la misma especie son una de las vías más importantes para obtener felicidad (desde nuestra perspectiva, placer básico y sobre todo disfrute complejo) y proba- 
blemente una de las vías más complejas y desconocidas. Puede concluirse que las vías y formas de obtener placer y sobre todo disfrute complejo son muchas y muy variadas, por ello tratar de hacer un listado con todas las circunstancias o situaciones susceptibles de obtener disfrute es una tarea casi imposible, y su evaluación sería muy larga y pesada por parte del evaluado. Un ejemplo de ello fue el listado con 320 potenciales reforzadores propuesto por MacPhillamy y Lewinsohn (1982).

La mayoría de instrumentos de evaluación del disfrute o capacidad hedónica se han realizado desde el ámbito de la psicopatología, y concretamente con la intención de evaluar la anhedonia, concepto propuesto por Ribot (1897), que se define como la incapacidad de obtener placer o disfrute en diferentes circunstancias o realizando actividades que antes resultaban gratificantes.

La presencia de anhedonia en la infancia y adolescencia es frecuente en los cuadros de depresión mayor, especialmente en los más severos (Gabbay, Johnson, Alonso, Evans, Babb \& Klein, 2015). Se ha observado que las personas con depresión manifiestan una respuesta emocional positiva atenuada ante estímulos positivos comparados con controles sanos (Bylsma, Morris \& Rottenberg, 2008). También la anhedonia es un síntoma frecuente en el trastorno de estrés postraumático en niños (Thabet \& Vostanis, 2000).

Sin olvidar que la anhedonia se ha relacionado con la ideación e intento suicida de forma consistente, incluso de forma independiente a la sintomatología depresiva (Winer, Drapeau, Veilleux \& Nadorff, 2016).

Los instrumentos para evaluar la anhedonia se han desarrollado para detectar la presencia de patología, originalmente y de forma más frecuente, la esquizofrenia. Uno de los instrumentos más conocidos son las escalas para la evaluación de la anhedonia física y social de Chapman, Chapman y Raulin (1976). Otro instrumento para evaluar la anhednonia y que también se ha utilizado frecuentemente para evaluar a pacientes psiquiátricos es la escala de placer de Fawcett, Clark, Scheftner y Gibbons (1983). Para la detección de la anhedonia en trastornos afectivos, se utiliza frecuentemente la escala de placer de Snaith-Hamilton (Snaith, et al., 1995). También existe un instrumento para evaluar a personas que se han expuesto a circunstancias de gran peligro o riesgo para su integridad física o moral, con riesgo de padecer un trastorno por estrés postraumático, se trata de la escala de interferencia y déficit hedónico de Frewen, Dean y Lanius (2012). Como se ha comentado 
arriba, todos han sido diseñados para detectar patología y hasta la fecha no se ha encontrado ninguno para ser aplicado en población infanto-juvenil.

Es por ello que el objetivo de esta investigación fue construir y estudiar las propiedades psicométricas de una Escala para medir el Disfrute Experimentado en Niños y Adolescentes (EDENA).

\section{MÉTODO}

\subsection{Diseño}

La presente investigación es de tipo psicométrico y por ello instrumental, ya que tiene como objetivo desarrollar pruebas y aparatos, que incluye la elaboración o adaptación de escalas y el estudio de sus propiedades psicométricas (Ato, López-García \& Benavente, 2013).

\subsection{Participantes}

Se tomó una muestra de 487 alumnos, los cuales 226 (46,4\%) eran de género masculino y 261 (53,6\%), del femenino de diferentes escuelas de secundaria de la ciudad de Morelia (Mich, México) de edades que iban desde los 11 hasta los 15 años de edad (con una media de 13,21 años y una desviación típica de 1,36 años). Los participantes estaban cursando; primero 50 alumnos $(10,3 \%)$, segundo 164 alumnos $(33,7 \%)$ y tercer grado 273 alumnos $(56,1 \%)$ de secundaria, los cuales accedieron voluntariamente a participar en el estudio, después de que los padres (o tutores) firmaran un consentimiento informado. El muestreo realizado fue de tipo no aleatorio y por conveniencia.

\subsection{Instrumentos}

\subsubsection{Escala de Disfrute Experimentado para Niños y Adolescentes} (EDENA)

Objeto de estudio de la presente investigación, para evaluar el disfrute experimentado. Con reactivos como "Disfruto durante muchos momentos al día" o "Soy una persona que disfruta con facilidad". Consta de siete reactivos con tres opciones de respuesta ("Totalmente de acuerdo" (2), "Ni en acuerdo ni en desacuerdo" (1) y "Nada de acuerdo" (0)). Se estudió psicométricamente la escala de siete reactivos aprobada por los jueces (ver procedimiento).

\subsubsection{Escalas PANASN (Positive And Negative Affect Schedule)}

Fue elaborada por Sandín (2003) para niños y adolescentes a partir de la forma para adultos PANAS de Watson, Clark y Tellegen (1988). EI PANASN al 
igual que el PANAS, es un cuestionario de autoinforme de 20 elementos, 10 ítems evalúan el afecto positivo (p.ej: "Soy una persona animada" o "Suelo emocionarme") y otros 10 de afecto negativo (p.ej: "Me siento nervioso" o "Me siento culpable"). El cuestionario es cumplimentado por el niño/adolescente teniendo en cuenta la manera en que este se siente y/o comporta habitualmente, siguiendo una escala de tres alternativas respuestas, descritas como: Nunca (1), A veces (2) y Muchas veces (3). La interpretación de las puntuaciones totales, del nivel de afecto positivo y afecto negativo son: a mayor puntuación indica que el evaluado experimenta dicho tipo de afecto con mayor frecuencia.

Las escalas PANASN tienen una consistencia interna que no alcanza el valor de alfa de Cronbach de 0,80 pero todos los coeficientes están por encima de 0,70 (Sandín, 2003).

\subsubsection{Escala de Depresión del Centro de Estudios Epidemiológicos CES-D (Center of Epidemiological Studies of Depression)}

Desarrollada por Radloff (1977) y adaptada a población mexicana (incluida la población de Michoacán) por Jiménez-Tapia, Wagner, Rivera-Heredia y González-Forteza (2015). Esta escala evalúa la sintomatología depresiva presente en la semana previa a la aplicación a través de 20 ítems. En México ha mostrado ser un instrumento adecuado para medir la sintomatología depresiva en adolescentes estudiantes. La consistencia interna de la escala alcanza valores iguales o superiores a .84 (alfa de Cronbach). Consta de cuatro factores que explican más del $52 \%$ de la varianza total. Se interpreta de modo que a mayor puntuación de la escala total supone que el evaluado presenta mayor sintomatología depresiva.

\subsection{Procedimiento}

Esta investigación se llevó a cabo en dos fases, en la primera se redactaron 11 reactivos con base en el constructo de disfrute. Después dos jueces evaluaron la pertinencia de cada uno de los reactivos con la definición operativa del concepto (revisaron los reactivos, teniendo presente si realmente estaban evaluando el disfrute experimentado en alguna de las tres modalidades: intensidad, frecuencia o duración). Después de dicha fase, se consideraron 7 ítems, aprobados por los jueces, que valoraron con una calificación subjetiva de 8 o superior (en una escala de 0 al 10) la pertinencia de cada reactivo para integrar la escala. 
En la segunda fase, se solicitó permiso a la dirección de las diferentes escuelas de secundaria de la ciudad de Morelia (Mich, México) para la aplicación de las escalas EDENA, CES-D y las PANASN a los alumnos de primero, segundo y tercero de secundaria. La aplicación de las escalas fue de manera grupal anónima y voluntaria y duró entre 25 y 30 minutos por grupo, El aplicador llegó al aula, se presentó con el grupo, entregó uno a uno y de manera individual las escalas y les pidió a cada uno de los participantes aportar la máxima atención y contestar todos los ítems sin excepción, el aplicador permaneció en el aula para controlar que se realizara de manera individual y para aclarar las dudas durante la aplicación. Al finalizar, cada uno de los participantes entregó la prueba al aplicador, quien les agradeció por su participación. Al obtener las pruebas contestadas los datos extraídos fueron capturados en la base de datos del programa SPSS versión 17.0 para hacer el estudio estadístico y obtener las propiedades psicométricas.

\subsection{Análisis de datos}

Se realizó un análisis factorial exploratorio a través del método de extracción de componentes principales con rotación de tipo Oblimin. La consistencia interna se estudió mediante el índice alfa de Cronbach. Se utilizó la $r$ de Pearson y los descriptivos media y desviación típica.

\subsection{Conflictos de interés}

Los autores del artículo declaran que no existe ningún conflicto de intereses con este trabajo.

\section{RESULTADOS}

Un total de 479 (98,36\%) participantes completaron las escala EDENA, y 8 $(1,64 \%)$ no respondieron a la totalidad de los reactivos.

\subsection{Validez de contenido}

Después de la revisión sobre la congruencia del contenido de los reactivos con el constructo, 7 ítems fueron aprobados por tres jueces, que valoraron con una calificación subjetiva de 8 o superior (en una escala de 0 al 10) la pertinencia de cada reactivo para integrar la escala.

\subsection{Estructura interna}

Respecto a la estructura interna, se realizó un análisis factorial exploratorio a través del método de extracción de componentes principales con rotación 
de tipo Oblimin. Previamente, se observó que los datos eran adecuados para dicho análisis (Test de esfericidad de Bartlett $=325.344 ; \mathrm{gl}=21$ con $\mathrm{p}$ $<0,001$; índice Kaiser Meyer Olkin =0,775). Se consideró la solución de dos factores (debido a que dos factores obtenían un valor propio superior a 1), sin embargo, esta solución no resultó satisfactoria (puesto que el segundo factor solo contenía 2 reactivos, y resultó difícil de interpretar, ya que uno alude a frecuencia y el otro a intensidad), por lo cual se eliminaron los reactivos 1 y 4 (ver Tabla 1).

Tabla 1.

Matriz de configuración bifactorial 7 ítems

\begin{tabular}{ccc}
\hline \multicolumn{2}{c}{ MATRIZ DE ESTRUCTURA } & \\
\hline Reactivo & 1 & 2 \\
1.-Me entretengo frecuentemente & $-0,038$ & 0,802 \\
2.-Me la paso bien en muchos lugares & 0,703 & 0,058 \\
3.-Disfruto durante muchos momentos del día & 0,648 & 0,237 \\
4.-Me divierto con mucha intensidad & 0,330 & 0,619 \\
5.-Estoy a gusto con la mayoría de las personas & 0,617 & $-0,043$ \\
6.-Me la paso bien en la mayoría de las circunstancias & 0,663 & 0,188 \\
7.-Soy una persona que disfruta con facilidad & 0,565 & 0,467 \\
\hline
\end{tabular}

Después se contempló la idoneidad de la solución de un solo factor, con los cinco reactivos restantes. Se observó que un factor explicaba casi el $42 \%$ de la varianza, además de una gran diferencia entre el valor propio del primer factor (el único superior a la unidad) y los otros. Por otro lado, todos los reactivos manifestaron una adecuada carga factorial (ver tablas 2 y 3 ), por ello se concluye que es una escala unifactorial.

Tabla 2.

Valores propios, varianza explicada y acumulada de los factores del EDENA

\begin{tabular}{cccc}
\hline COMPONENTE & AUTOVALOR & $\begin{array}{c}\text { \% VARIANZA } \\
\text { EXPLICADA }\end{array}$ & $\begin{array}{c}\text { \% VARIANZA ACUMULADA } \\
\text { EXPLICADA }\end{array}$ \\
\hline 01 & 2,097 & 41.938 & 41.938 \\
02 & 0,805 & 16.108 & 58.047 \\
03 & 0,729 & 14.584 & 72.630 \\
04 & 0,698 & 13.961 & 86.592 \\
05 & 0,670 & 13.408 & 100.000 \\
\hline
\end{tabular}


Tabla 3.

Cargas factoriales de los cinco reactivos de la EDENA (con los cinco reactivos de la versión final).

\begin{tabular}{cc}
\hline ÍTEMS & FACTOR ÚNICO \\
\hline 1 & 0,684 \\
2 & 0,668 \\
3 & 0,583 \\
4 & 0,669 \\
5 & 0,629 \\
\hline
\end{tabular}

\subsection{Confiabilidad y Bondad de los reactivos}

Respecto a la consistencia interna, el valor del alfa de Cronbach de la escala resultó de .650 , aceptable teniendo en cuenta que solo tiene cinco reactivos. De forma paralela, se estudió la bondad de los reactivos, los valores de la desviación típica y la media de los reactivos oscilaron entre 0,49 a 0,56 y 1,43 a 1,68 respectivamente, lo cual muestra que el promedio de cada uno de los reactivos está por encima de la media teórica, considerando que los valores de respuesta oscilaban de 0 a 2 . Los reactivos manifestaron una moderada correlación con la puntuación total de escala corregida (excluyendo a los propios ítems) que osciló de .351 a .438 , y si algún reactivo es eliminado el valor del alfa total de la escala disminuye considerablemente (ver Tabla 4).

Tabla 4.

Media, desviación estándar, correlación del ítem con la escala total (corregida) y el valor del alfa de Cronbach si se elimina el ítem de los reactivos del EDENA

\begin{tabular}{ccccc}
\hline ÍtEMS & MEDIA & $\begin{array}{c}\text { DESVIACIÓN } \\
\text { TíPICA }\end{array}$ & $\begin{array}{c}\text { CORRELACIÓN } \\
\text { ITEM ESCALA TOTAL } \\
\text { (CORREGIDA) }\end{array}$ & $\begin{array}{c}\text { ALFA DE LA ESCALA } \\
\text { TOTAL SI SE ELIMINA } \\
\text { ÍTEM }\end{array}$ \\
\hline 1 & 1,65 & 0,50 & 0,438 & 0,582 \\
2 & 1,68 & 0,49 & 0,422 & 0,590 \\
3 & 1,51 & 0,56 & 0,351 & 0,624 \\
4 & 1,43 & 0,54 & 0,426 & 0,587 \\
5 & 1,65 & 0,53 & 0,385 & 0,606 \\
\hline
\end{tabular}

\subsection{Relación con otras variables y Validez concurrente}

Con el objetivo de obtener las primeras estimaciones de evidencias de validez concurrente de la escala, se realizaron correlaciones de Pearson entre la escala EDENA y las escalas PANAS y CES-D. La correlación resultó baja y positiva con la escala de Afecto Positivo $(r=.368)$, baja y negativa con el Afecto Negativo ( $r=-.361)$, y muy baja, negativa $(r=-.179)$, pero también 
significativa con la escala de depresión (CES-D), todas las correlaciones con $\mathrm{p}<0.001)$.

\subsection{Datos descriptivos y relación con edad y sexo}

Por otro lado, se observó que la media del EDENA resultó de 7,91 (DE =1,69), los valores más frecuentes fueron el 8 y el 9 , el valor mínimo fue de 0 y el máximo de 10. Véanse las frecuencias en la Tabla 5.

Tabla 5.

Frecuencias y porcentajes (simples y acumulados) de los valores de la puntuación total de la escala EDENA

\begin{tabular}{ccccc}
\hline VALORES & FRECUENCIA & $\%$ & $\begin{array}{r}\text { FRECUENCIA } \\
\text { ACUMULADA }\end{array}$ & $\begin{array}{c}\text { PORCENTAJE } \\
\text { ACUMULADO }\end{array}$ \\
\hline 0 & 1 & 0,2 & 1 & 0,2 \\
3 & 8 & 1,6 & 9 & 1,9 \\
4 & 8 & 1,6 & 17 & 3,5 \\
5 & 24 & 4,9 & 41 & 8,6 \\
6 & 50 & 10,3 & 91 & 19,0 \\
7 & 85 & 17,5 & 176 & 36,7 \\
8 & 104 & 21,4 & 280 & 58,5 \\
9 & 104 & 21,4 & 384 & 80,2 \\
10 & 95 & 19,5 & 479 & 100,0 \\
\hline
\end{tabular}

Respecto al estudio de posibles diferencias en función del sexo, se observó que la media de las participantes femeninas $(M=7,88, D E=1,68)$ no difería $(t=0,482 ; p=.630)$ de la obtenida por los participantes masculinos $(M=7,95, D E=1,71)$. Finalmente, respecto a la relación entre edad y disfrute experimentado se obtuvo una correlación baja, negativa y significativa $(r=-.172 ; p<.001)$.

\section{DISCUSIÓN}

El objetivo principal de este trabajo fue construir y estudiar las propiedades psicométricas de una escala para valorar y medir el disfrute experimentado en niños y adolescentes. Tras un análisis factorial exploratorio se concluyó que la mejor solución era la unifactorial, y quedó constituida por cinco ítems que pesaban de forma considerable en el único factor. El hecho de hallar una sola dimensión podría ser consecuencia de que en la presente escala se han planteado preguntas generales acerca del disfrute obtenido, y no se han contemplado modalidades, es posible que si se considerara en la redacción de los reactivos diferentes modalidades (el saborear, el disfrute realizando 
ejercicio, en relaciones interpersonales, etc.) probablemente se hallaría una estructura multifactorial.

La confiabilidad de la escala puede considerarse aceptable debido al reducido número de reactivos. Los cinco reactivos que constituyen la escala resultaron adecuados, teniendo como criterio la correlación con la escala total (excepto el propio ítem) y el impacto en la consistencia interna del total de la escala.

Se estudió la validez concurrente de la Escala de Disfrute Experimentado con la Escala de Afecto Positivo y Afecto Negativo para Niños (PANASN) y la Escala de Depresión del Centro de Estudios Epidemiológicos (CES-D). Se obtuvo una correlación baja y positiva con la escala de Afecto Positivo, en principio, se esperaba obtener una correlación algo más elevada. Después de una revisión sobre el contenido de los reactivos, pensamos que la correlación baja obtenida podría deberse a que solo dos (de diez) reactivos de la escala de afecto positivo de la escala PANASN están relacionados con el disfrute $\left(3^{\circ}\right.$ "Soy una persona animada, suelo emocionarme" y $9^{\circ}$ "Me entusiasmo por cosas personales", etc.) mientras que el resto se relacionan más con la atención ( $17^{\circ}$ "Soy una persona atenta, esmerada"), el orgullo ( $5^{\circ}$ "Me siento orgulloso/a (de algo), satisfecho/a"), la inspiración (14 "Me siento inspirado/a") y qué tan activos son (19' "Soy un/a chico/a activo/a"), por mencionar algunos y resaltar que ningún reactivo pregunta literalmente por el disfrute.

Respecto a la escala de afecto negativo y la escala de depresión se obtuvieron correlaciones bajas y negativas las cuales eran esperadas. En cuanto a la relación con la sintomatología depresiva debe recordarse que frecuentemente en los trastornos depresivos unipolares aparece el síntoma de disminución importante del interés o disfrute por todas o casi todas las actividades la mayor parte del día, casi todos los días (información que se desprende de la apreciación subjetiva o de la observación) como aparece en el manual diagnóstico del DSM-5 (APA, 2013). Sin embargo, debe recordarse que para realizar el diagnóstico de trastorno depresivo mayor no es necesario cumplir con el criterio de pérdida de interés o placer, debe cumplir un mínimo de cinco (por ejemplo: estado de ánimo deprimido, alteraciones del sueño, del peso, del movimiento, presencia de fatiga, etc.) criterios de nueve (APA, 2013), lo cual explica la baja correlación con la sintomatología depresiva. Sobre la baja correlación con el afecto negativo, debe recordarse que cuando se alude a las emociones, aparece ajustarse un modelo bidimensional unifactorial PadrósBlázquez et al. (2012), lo cual explica la baja correlación hallada. 
Se realizó un análisis entre sexos para saber si había diferencias estadísticamente significativas entre niños y niñas. Los resultados hallados no muestran diferencias en cuanto al nivel de disfrute experimentado, esto puede ser debido a que están en una etapa donde se inicia la aparición de los caracteres sexuales secundarios, los roles todavía no están muy definidos, y probablemente el inicio de la adolescencia incida en gran medida en el estado afectivo pero independientemente del sexo. En adición a ello, en otras investigaciones se ha encontrado que no existen diferencias significativas en el afecto positivo entre niños y niñas (Sandín, 2003). Por otro lado, se observó que a mayor edad en los participantes, peor nivel de disfrute experimentado obtenían (aunque una relación muy baja), lo cual es congruente, ya que los periodos críticos de los adolescentes en edades de 14 o 15 suelen ser más intensos que los experimentados durante los 11 o 12 años. Por otro lado, parece que el autoconocimiento emocional no tendría por qué relacionarse con el disfrute experimentado, ya que en el estudio de Santoya Montes, Garcés Prettel y Tezón Boutureira (2018) se obtuvo una correlación positiva entre edad y autoconocimiento. Sin embargo, no se han hallado datos de otros investigadores acerca de los niveles de afecto positivo en relación con la edad en niños y adolescentes.

En el presente trabajo se identificaron algunas limitaciones importantes, la más notable es que no existe otra escala que evalúe el disfrute experimentado, por consiguiente no existe un parámetro donde los investigadores puedan medir la validez del instrumento (a modo de "Gold estándar"). Podría utilizarse en un futuro alguna medición de respuesta a diferentes potenciales reforzadores como la propuesta por MacPhillamy y Lewinsohn (1982), aunque sugeriríamos que se utilizara una versión reducida. Por otro lado, debe mencionarse que la escala PANASN aún no está estandarizada en población mexicana.

Por otro lado, las correlaciones mostradas por el EDENA con las escalas PANASN y CES-D muestran indicios de una adecuada validez concurrente de la escala. Sin embargo, sería conveniente hacer uso de otras escalas que midieran las misma u otras variables (por ejemplo: impulsividad, satisfacción con la vida, autoestima, etc.) para corroborar la validez del instrumento. Asimismo, sería pertinente comprobar la estructura unifactorial de la escala a través de un Análisis Factorial Confirmatorio.

También se sugiere para futuras investigaciones estudiar la fiabilidad test-retest. Asimismo, sería interesante ampliar la muestra incluyendo población con características sociodemográficas más variadas y estudiar su relación con el disfrute experimentado. Podría ser interesante estudiar la sensibilidad al cambio en niños o adolescentes con trastornos afectivos, o experiencias de abuso, 
traumáticas, etc. que mostraran una mejoría clínica significativa después de recibir un tratamiento.

En la valoración de las puntuaciones totales se observa que hasta un 8,6 \% de los evaluados muestra una puntuación igual o inferior a 5, ello podría ser un indicio de riesgo (o quizás 4 o 6), teniendo presente que podría ser un indicador de presencia de anhedonia, que se asocia con disfunción social y emocional, además de psicopatología (Dodell-Feder \& Germine, 2018). Como señalan Husain y Roiser (2018) puede considerarse la anhedonia como una aproximación transdiagnóstica. Sería interesante en un futuro, realizar investigaciones con la intención de hallar algún punto de corte que indicara riesgo de disfunción social, emocional o de psicopatología.

A modo de conclusión, deseamos señalar que tomando en consideración los resultados de la presente investigación se puede concebir a la escala EDENA, como un instrumento psicométrico con aceptables propiedades para población de Michoacán (México).

Financiamiento: El presente trabajo estuvo financiado por la Coordinación de Investigación Científica de la Universidad Michoacana de San Nicolás de Hidalgo dentro del proyecto "Estudio psicométrico de la Escala de Gaudibilidad para Niños y Adolescentes (EGNA) de población mexicana".

Agradecimientos: A los directores, profesores y alumnos de las diferentes escuelas de secundaria de la ciudad de Morelia (Mich, México) que nos permitieron amablemente y de forma desinteresada realizar la aplicación de las escalas.

\section{REFERENCIAS}

Alcántara, G. (2008). La definición de la salud de la Organización Mundial de la Salud y la interdisciplinariedad. Sapiens, 9(1), 93-107. http://132.248.9.34/hevila/ Sapiens/2008/vol9/no1/4.pdf

Allman, J.M. (1999). Evolving brains. New York: Scientific American Library. https:// authors.library.caltech.edu/25078/1/Evolving_Brains.pdf

Allport, G. (1961). Pattern and growth in personality. New York: Holt, Rinehart and Winston, Inc.

American Psychiatric Association (2013). Diagnostic and statistical manual of mental disorders (5th ed.). Washington, DC: Autor. https://doi.org/10.1176/appi. books.9780890425596

Ato, M., López-García, J. J., \& Benavente, A. (2013). Un sistema de clasificación de los diseños de investigación en psicología. Anales de Psicología, 29(3), 1038-1059. https://doi.org/10.6018/analesps.29.3.178511

Bustos, V.A. \& Russo, A.R. (2018). Salud mental como efecto del desarrollo psicoafectivo en la infancia. Psicogente, 21(39), 183-202. http://doi.org/10.17081/ psico.21.39.2830 
Bylsma, L. M., Morris, B. H., \& Rottenberg, J. (2008). A meta-analysis of emotional reactivity in major depressive disorder. Clinical Psychology Review, 28(4), 676-691. http://dx.doi.org/10.1016/j.cpr.2007.10.001

Castro, A. (2010). Fundamentos de psicología positiva. Argentina: Editorial Paidós.

Chapman, L. J., Chapman, J. P., \& Raulin, M. L. (1976). Scales for physical and social anhedonia. Journal of abnormal psychology, 85(4), 374-382. https://www.ncbi. nlm.nih.gov/pubmed/956504

Davis, W. A. (1982). A causal theory of enjoyment. Mind, 91(362), 240-256. https://doi. org/10.1093/mind/XCl.362.240

Diener, E. (1984). Subjective Well-being. Psychological Bulletin, 95, 542-575. http:// dx.doi.org/10.1037/0033-2909.95.3.542

Dodell-Feder, D., \& Germine, L. (2018). Epidemiological Dimensions of Social Anhedonia. Clinical Psychological Science, 1-9. http://dx.doi.org/10.1177/2167702618773740

Fawcett, J., Clark, D. C., Scheftner, W. A., \& Gibbons, R. D. (1983). Assessing anhedonia in psychiatric patients: The Pleasure Scale. Archives of general psychiatry, 40(1), 79-84. http://dx.doi.org/10.1001/archpsyc.1983.01790010081010

Frewen, P. A., Dean, J. A., \& Lanius, R. A. (2012). Assessment of anhedonia in psychological trauma: development of the Hedonic Deficit and Interference Scale. European Journal of Psychotraumatology, 3(1), 8585. https://doi.org/10.3402/ejpt.v3i0.8585

Gabbay, V., Johnson, A. R., Alonso, C. M., Evans, L. K., Babb, J. S., \& Klein, R. G. (2015). Anhedonia, but not irritability, is associated with illness severity outcomes in adolescent major depression. Journal of child and adolescent psychopharmacology, 25(3), 194-200. https://doi.org/10.1089/cap.2014.0105

Husain, M., \& Roiser, J. P. (2018). Neuroscience of apathy and anhedonia: a transdiagnostic approach. Nature Reviews Neuroscience, 19(8), 1.https://doi.org/10.1038/ s41583-018-0029-9

Jiménez-Tapia, A., Wagner, F., Rivera-Heredia, M. E., \& González-Forteza, C. (2015). Estudio de la depresión en estudiantes de la Ciudad de México y del Estado de Michoacán por medio de la versión revisada de la CES-D. Salud Mental, 38(2), 103-107. https://doi.org/10.17711/SM.0185-3325.2015.014

Keyes, C., Ryff, C., \& Shmotkin, D. (2002). Optimizing well-being: the empirical encounter of two traditions. Journal of personality and social psychology, 82, 1007-1022. http://dx.doi.org/10.1037/0022-3514.82.6.1007

Kimiecik, J. \& Harris, A.T. (1996). What is enjoyment? A conceptual/definitional analysis with implications for sport and exercise psychology. Journal of Sport \& Exercise Psychology, 18, 4-8. https://doi.org/10.1123/jsep.20.3.247

Kringelbach, M. L., y Berridge, K. C. (2009). Towards a functional neuroanatomy of pleasure and happiness. Trends in cognitive sciences, 13(11), 479-487. https://doi. org/10.1016/j.tics.2009.08.006

Kringelbach, M. L., \& Berridge, K. C. (2010a). The functional neuroanatomy of pleasure and happiness. Discovery medicine, 9(49), 579-587. https://www.ncbi.nlm.nih.gov/ pmc/articles/PMC3008353/

Kringelbach, M. L., \& Berridge, K. C. (2010b). The neuroscience of happiness and pleasure. Social Research, 77(2), 659. https://www.ncbi.nlm.nih.gov/pmc/articles/ PMC3008658/

MacPhillamy, D. J., \& Lewinsohn, P. M. (1982). The pleasant events schedule: Studies on reliability, validity, and scale intercorrelation. Journal of Consulting and Clinical Psychology, 50(3), 363-380. http://dx.doi.org/10.1037/0022-006X.50.3.363 
Maslow, A. (1968) Towards a Psychology of being. New York: D. van Norstrand. (Trad. cast: El hombre autorrealizado: hacia una psicología del ser. Barcelona: Kairós, 1998, 1a ed).

Padrós, F. (2002). Disfrute y bienestar subjetivo un estudio psicométrico de la gaudibilidad. (Tesis Doctoral Universidad de Barcelona. Barcelona, España. http://www. tesisenred.net/bitstream/handle/10803/4741/fpb1de2.pdf?sequence=1

Padrós-Blázquez, F., Contreras-Navarro, G. \& Soriano-Más C. (2012). Afecto positivo y negativo: ¿una dimensión bipolar o dos dimensiones unipolares independientes? Interdisciplinaria y Ciencias Afines, 29(1), 1-14. http://dx.doi.org/10.16888/ interd.2012.29.1.9

Radloff, L. S. (1977). The CES-D scale: A self-report depression scale for research in the general population. Applied psychological measurement, 1(3), 385-401. https:// doi.org/10.1177/014662167700100306

Ribot, T. (1897). The psychology of emotions. Londres: W. Scott Pub. Co. https://archive. org/details/psychologyofemo00ribo

Rogers, C. (1961). On becoming a person. Boston, M.A: Houghton Mifflin. (Trad. cast: EI proceso de convertirse en persona. Barcelona: Paidós, 2004). http://s-f-walker.org. uk/pubsebooks/pdfs/Carl_Rogers_On_Becoming_a_Person.pdf

Ryan, R.M., \& Deci, E.L. (2001). On happiness and human potentials: A review of research on hedonic and eudaimonic well-being. Annual review of psychology, 52, 141-166. https://doi.org/10.1146/annurev.psych.52.1.141

Sandín, B. (2003). Escalas PANAS de Afecto Positivo y Negativo para Niños y Adolescentes (PANASN). Revista de Psicopatología y Psicología Clínica, 8(2), 173-182. https://doi.org/10.5944/rppc.vol.8.num.2.2003.3953

Santoya Montes, Y., Garcés Prettel, M. \& Tezón Boutureira, M. (2018). Las emociones en la vida universitaria: análisis de la relación entre autoconocimiento emocional y autorregulación emocional en adolescentes y jóvenes universitarios. Psicogente, 21(40), 422-439. https://doi.org/10.17081/psico.21.40.3081

Scanlan, T. K., \& Simons, J. P. (1992). The construct of sport enjoyment. In G. C. Aoberts (Ed.), Motivation In sport and exercise (pp. 199-215). Champaign, IL: Human Kinetics.

Seligman, M.P. \& Csikszentmihalyi, M. (2000). Positive psychology: An introduction. American Psychologist, 55, 5-14. http://dx.doi.org/10.1037/0003-066X.55.1.5

Snaith, R. P., Hamilton, M., Morley, S., Humayan, A., Hargreaves, D., \& Trigwell, P. (1995). A scale for the assessment of hedonic tone the Snaith-Hamilton Pleasure Scale. The British Journal of Psychiatry, 167(1), 99-103. https://doi.org/10.1192/bjp.167.1.99

Thabet, A. A., \& Vostanis, P. (2000). Post-traumatic stress disorder reactions in children of war: a longitudinal study. Child Abuse \& Neglect, 24(2), 291-298. https://doi. org/10.1016/S0145-2134(99)00127-1

Veenhoven, R. (1994). El estudio de la satisfacción con la vida. Intervención Psicosocial, 3, 87-116. https://personal.eur.nl/veenhoven/Pub1990s/94d-fulls.pdf

Watson, D., Clark, L. A., \& Tellegen, A. (1988). Development and validation of brief measures of positive and negative affect: the PANAS scales. Journal of Personality and Social Psychology, 54(6), 1063-1070. http://dx.doi.org/10.1037/00223514.54.6.1063

Winer, E. S., Drapeau, C. W., Veilleux, J. C., \& Nadorff, M. R. (2016). The association between anhedonia, suicidal ideation, and suicide attempts in a large student sample. Archives of Suicide Research, 20(2), 265-272. https://doi.org/10.1080/13 811118.2015.1025119 


\section{ANEXO 1. \\ Escala de Disfrute Experimentado para Niños y Adolescentes (EDENA)}

\begin{tabular}{|c|c|c|c|}
\hline $\begin{array}{l}\text { SEÑALA EL NIVEL DE ACUERDO EN CADA UNA DE } \\
\text { LAS SIGUIENTES AFIRMACIONES: }\end{array}$ & $\begin{array}{l}\text { TOTALMENTE DE } \\
\text { ACUERDO }\end{array}$ & $\begin{array}{l}\text { NI EN ACUERDO NI } \\
\text { EN DESACUERDO }\end{array}$ & $\begin{array}{l}\text { NADA DE } \\
\text { ACUERDO }\end{array}$ \\
\hline 1. Me lo paso bien en muchos lugares. & (3) & $(\ddot{\theta})$ & \\
\hline 2. Disfruto durante muchos momentos al día. & $(0)$ & 9 & (2) \\
\hline 3. Estoy a gusto con la mayoría de personas. & (3) & $\underline{\theta}$ & (8) \\
\hline 4. Me lo paso bien en la mayoría de circunstancias. & (9) & $\underline{\theta}$ & (8) \\
\hline 5. Soy una persona que disfruta con facilidad. & (3) & $(\ddot{\theta})$ & () \\
\hline
\end{tabular}

Esta obra está bajo: Creative commons attribution 4.0 international license. El beneficiario de la licencia tiene el derecho de copiar, distribuir, exhibir y representar la obra y hacer obras derivadas siempre y cuando reconozca y cite la obra de la forma especificada por el autor o el licenciante.

\section{(cc) BY}


